\title{
A simple projection photolithography method for low-cost rapid prototyping of microfluidic chips
}

\author{
Stefan Ostmann ${ }^{1} \cdot$ Christian J. Kähler ${ }^{1}$
}

Received: 13 September 2021 / Accepted: 13 February 2022 / Published online: 3 March 2022

(c) The Author(s) 2022

\begin{abstract}
Presented herein is a masking method for SU8 based soft lithography which utilizes a simple optical setup using transparencies printed with a commercial standard office printer. The relatively low printing quality of the printer is partly overcome by imaging of the mask with negative magnification that utilizes relatively cheap and easily available parts. The imaging quality as well as the quality of the resulting SU8 master is assessed by means of microscopic imaging.
\end{abstract}

\section{Introduction}

There are numerous applications for the use of microfluidics, such as for example hematology (Holmes et al. 2009), viscosimetry (Guillot et al. 2006) or interfacial tensiometry (Cabral and Hudson 2006) and the food industries (He et al. 2020). A particularly interesting field of application is in hand-held devices, which hold for example optical sensorics and use disposable microfluidic chips. Especially, with the use of recent developments, such as all-Silicon microdisplays (Wu et al. 2021) and on-chip microoptical sensors (Xu et al. 2019), which could offer high integration potential. However, the broad development of microfluidic application requires simple, fast and cost-effective ways to prototype geometries.

The soft lithography method has been arguably the most important and broadly used method for the production of microfluidic chips within the last decades. The process is basically composed of two steps. First, a rigid master is produced. It holds a negative of the desired geometry on a suitable substrate. In the second step, a polymer, commonly PDMS (Polydimethylsiloxane), is cast onto the negative structure. After the curing of the polymer, the master is removed, leaving behind the channel structure onto the polymer. Arguably, the key step of the soft lithography process is the selective exposure of a photoresist layer to UV radiation, which causes cross-linking of the polymer chains

Stefan Ostmann

stefan.ostmann@unibw.de

1 Institute for Fluid Mechanics and Aerodynamics, Bundeswehr University Munich, 85577 Neubiberg, Germany depending on the method used. In the next step the photoresist is developed by a suitable chemical that removes all noncross linked photoresist, leaving the negative of the desired microstructure. The most commonly used exposure method in microfluidics is the contact printing method. Here, the photomask is brought in direct contact with the photoresist, allowing for the passage of light only on a certain area. The optical resolution of the photomask determines the maximum achievable resolution of the microchannel. Therefore, the necessity of high optical resolution photo-masks is immediately understandable.

An alternative approach is called projection photo lithography (Rothschild 2005). In contrast to the contact printing methods, the pattern is not formed by the shadows of the photomask, but rather the image of the photomask is projected onto the substrate with optical reduction. The advantage of the used method is to be contact-less and comparatively flexible. In contrast to the contact printing method, the resolution of the photomask can be considerably lower, due to the optical reduction of the used optical system. This enables the use of commercial printers up to a certain degree of accuracy. A comparable approach was used by Love et al. (2001) reaching resolutions close to the refraction limit $(\sim 0.6 \mu \mathrm{m})$ using a commercial microscope, a mercury vapour lamp and film printed photomasks with 5000 dpi and immersion lenses to achieve a maximum resolutions close to the diffraction limit. The use of commercial unmodified microscopes however results in relatively small image-cross sections, which limits the achievable size of the actual geometry size to a cross section of $400 \mu \mathrm{m}$ (with $100 \times$ optical reduction). Kwon and Ju (2018) also used a commercial microscope, but with LED illumination, successfully 
producing refraction gratings. Wang et al. (2009) proposed a method using an DMD (digital micromirror device) which is able to produce SU8 Masters without the use of a photomask, where desired channel geometry could be projected onto the photoresist directly.

Especially in the field of microfluidics the producible geometry should be large enough to allow for connections to e.g. piping. Additionally, microfluidic structures often need non-negligible channel heights to limit the geometries pressure losses.

The herein proposed setup uses a large Fresnel lens as condenser lens and a microscopic lens objective (also the use of commercial achromatic lenses was tested). The setup enables the projection of a comparatively large area (low resolution) photomasks onto a comparatively large area of photoresist. This allows for the production of microfluidic geometries in one exposure step and offers a simple way to adjust the final size of the microchannels through adjustment of the optical reduction of the imaging system within certain boundaries. In this study we investigate the capabilities of the system, with regard to applicable optical reductions, necessary exposure times and obtainable image and mask cross sections. The aim of this paper is, to facilitate a low cost and easy to use tool for rapid prototyping of microfluidic geometries, enabling them to quickly find optimal geometries for research in the field of microfluidics.

\section{Materials and Methods}

The proposed setup uses an imaging system with an optical reduction with a system of two lenses, see Figs. 1 and 2). A Luminus CBM-120-UV-X31-I365 Power LED (actively

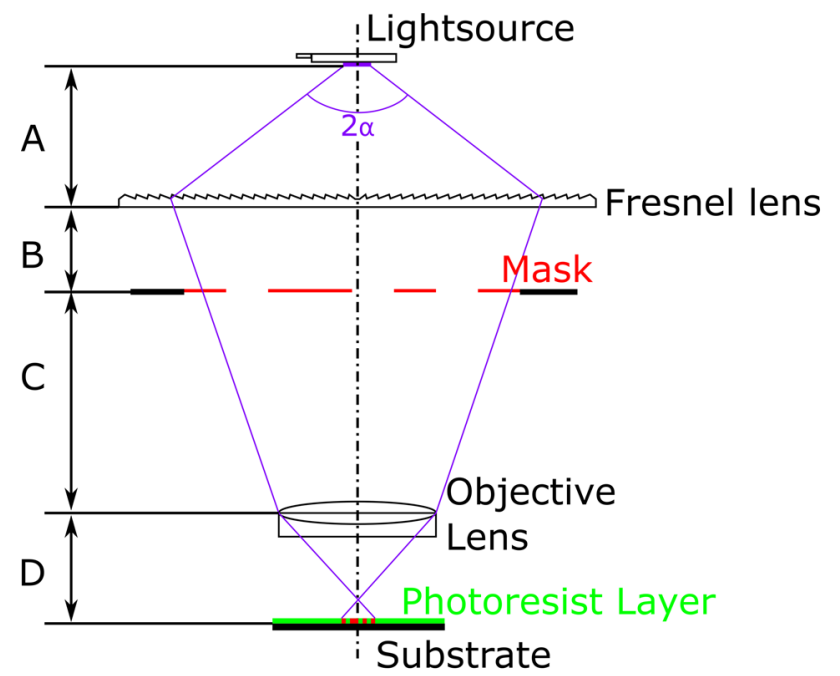

Fig. 1 Schematic setup with distances for $10 \times$ optical reduction A (255 mm), B (200 mm), C (930mm), D (130 mm)

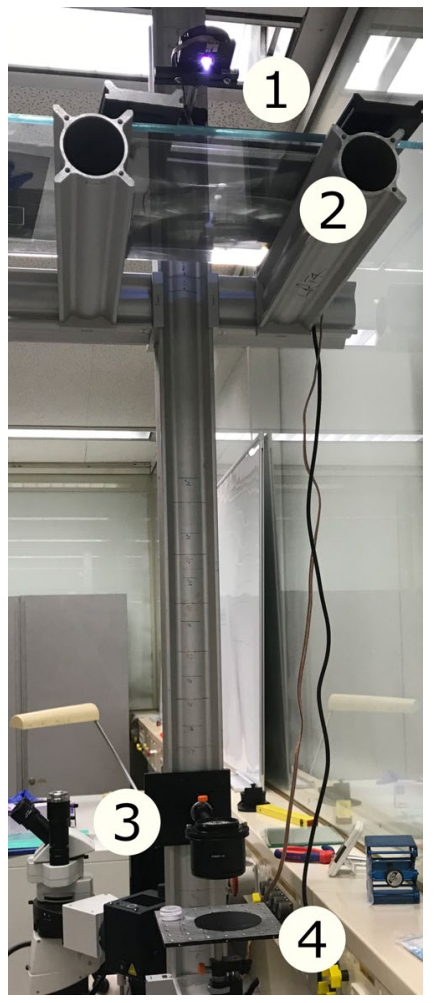

Fig. 2 (1): Light source (2): condenser and photomask (3): objective Lens (4): imaging plane and substrate

air cooled) light source with a peak wavelength of $365 \mathrm{~nm}$ was used. The LED was selected to match the main absorption peak of the photoresist. The lightsource was operated at constant wave (@12.7A, 5V).

The imaging is achieved with a lens-system consisting of two lenses (see Fig. 2). The first lens behind the light source is a Fresnel lens with a $500 \times 500 \mathrm{~mm}^{2}$ square cross section and a focal length of approximately $70 \mathrm{~mm}$. Fresnel lenses are generally known to have comparatively low imaging quality however, they present a good trade-off for illumination purposes because of their light weight, large area, low focal length and low costs. The large size of these kind of lenses, while possessing low focal length at the same time, allows for collimation of light over a large aperture. The Fresnel lens acts as condenser lens and makes the incident divergent rays of the light source convergent, focussing them on the second lens. The large area of the lens serves two purposes. First, the large area collects and collimates light of the divergent lightsource under an angle $\alpha \approx 40^{\circ}$, which involves light down to a relative intensity of $\approx 80 \%$ (Lum 2019) given the radiation pattern of the LED. Secondly, the photomask with an area of $297 \mathrm{~mm} \times 210 \mathrm{~mm}$ needs to be illuminated as completely and evenly as possible.

The second lens is the Imaging lens. In the presented study, a Leica $1 \times$ Planapochromat microscopic lens with 
an numerical aperture (NA) of 0.2 was used. The usage of a single lenses is also possible. However, for good imaging quality the Objective lens needs to have a good quality (achromats/duplets) and needs to correct for spherical aberrations. Additionally, the used microscope objective is optimized for high power UV throughput, thus originally built for florescence microscopy, and therefore causes low light absorbance in the UV spectrum.

Given the numerical aperture (NA) of the imaging lens and the object distance $\mathrm{C}$ (see Fig. 1 the resolution of the imaging system could be calculated via $b=\lambda /(2 N A)$, with $N A$ being the numerical aperture of the system and $\lambda$ the wavelength of the lightsource. Assuming, that the mask has a maximum dimension of $300 \mu \mathrm{m}$ and is positioned in the central the theoretical resolution is $\approx 1.1 \mu \mathrm{m}$ and could potentially be reduced by decreasing the object distance $C$. However this would lead to increasing losses in light intensity and and a less even illumination of the imaging plane.

The photomask is positioned onto an intermediate layer $150 \mathrm{~mm}$ below the Fresnel lens. The substrate, holding the photoresist, is positioned onto a vertically adjustable table. All components are positioned co-parallel and centred to the vertical (optical) axis. Apart from the condenser and the photomask, the vertical position of all optical elements is adjustable. However, due to the large distances between the optical elements, a relatively high uncertainty in their position adjustment was present. For the application of different optical reductions, herein defined as $M=X / x$ ( $X$ is the linear dimension on the photomask, $x$ is the linear dimension on the Image or the photoresist) the distances A, B and $\mathrm{C}$ (Fig. 1) have to be adjusted. The critical distance is the object distance $\mathrm{C}$. This distance varies the optical reduction ratio and determines the image size and system aperture. After setting distance B, distance A (distance between the condenser and the light source) has to be chosen in a way, that the aperture on the illumination side (lightsource, photomask and Fresnel lens) matches the aperture of the objective lens as close as possible. Finally the distance D is selected by adjusting the height of the substrate table. The optimum focus is set directly onto the surface of a test wafer. After the adjustment procedure, the setup is ready to be used for UV exposure of the photoresist. The photomask was printed onto a standard overhead transparency (A4, $210 \times 297 \mathrm{~mm}^{2}$, Acetate film) using an office laser printer with $1200 \mathrm{dpi}$. Figure 3 shows the geometry of the used grid and the resulting print quality. The test grid consists of a center dot, which is placed directly into the optical axis of the setup, as well as a small grid with $0.3 \mathrm{~mm}$ grid lines and a slightly larger grid with $0.5 \mathrm{~mm}$ linewidth. The grid spacing is $10 \mathrm{~mm}$ for both grids and the offset between the two grids is $5 \mathrm{~mm}$. The test grid geometry was produced using an open-source vector graphics tool. Figure 4 shows light microscope images of the section marked in Fig. 3. It can be seen that the printed

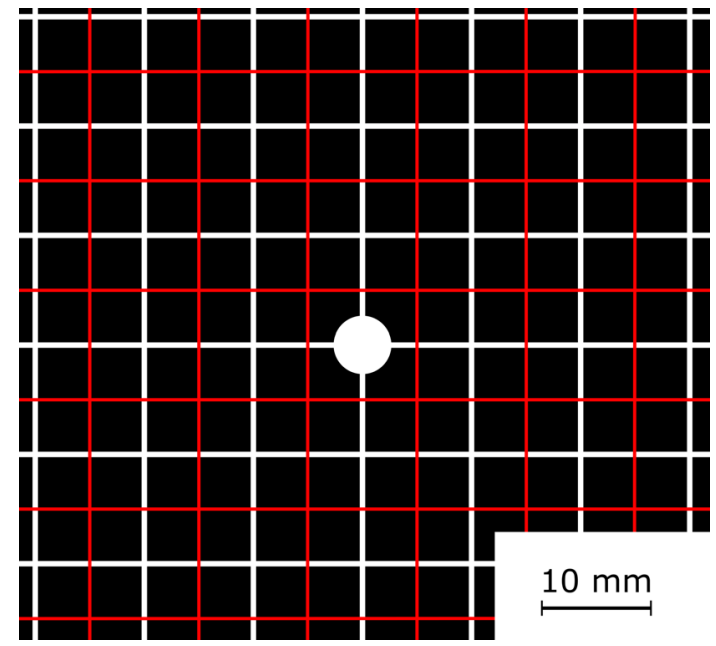

Fig. 3 Grid structure with center dot, small grid (red) and large grid (white) (color figure online)

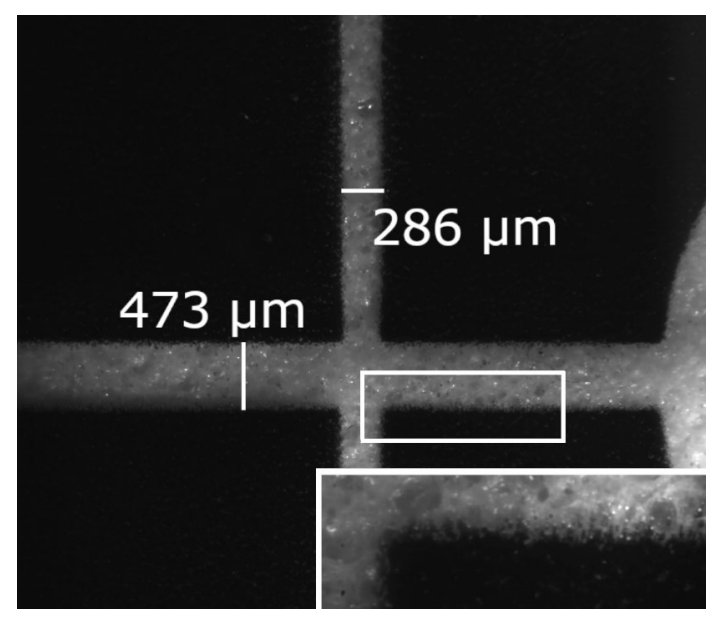

Fig. 4 Light microscopy image of the center mask section

films are accurate to about $\sim 40 \mu \mathrm{m}$. However, the prints are able to resemble the correct ratio between the two line widths of $0.5 / 0.3=1.66$. The transitional distance between the opaque the translucent sections are about $40 \mu \mathrm{m}$ (see Fig. 4). Considering the resolution $b$ of the optical system, it can be seen that the mask quality is the limiting factor of the proposed setup.

In the present study we used a photoresist by MICROCEM SU-2075. The substrate is a $100 \mathrm{~mm}$ standard silicon wafer. The photoresist layer thickness for all experiments was about $60 \mu \mathrm{m}$, which was produced by spin coating according to the data sheet. In the soft lithography process, a pre-exposure bake $\left(7 \mathrm{~min} @ 95^{\circ} \mathrm{C}\right)$ and a post-exposure bake $\left(10 \mathrm{~min} @ 95^{\circ} \mathrm{C}\right)$ was performed. The structures were developed using 1-Methoxy-2-Propylacetat (PGMEA, Sigma Aldrich) and Isopropanol as a rinse. The quality of 
the masters was investigated using a Keyence digital microscope at $100 \times$ magnification (pixel resolution $1 \mu \mathrm{m} /$ pixel). Additionally, the imaging quality for different optical reductions was priorly investigated with a CCD camera. The CCD camera chip (.pco4000, $2000 \times 4000$ pixel, $9 \mu \mathrm{m}$ pixel pitch) was positioned directly within the imaging plane of the setup. For this preliminary investigation the LED output power was reduced rigorously to avoid damaging the camera sensor.

The LED power was constant for all investigated optical reductions. Although, the camera is not able to quantitatively asses the irradiation, its pixel values are proportional to the radiometric flux (Hain et al. 2007) and therefore allow for a qualitative assessment of the uniformity of illumination. The illumination of the LED causes non-uniform illumination at the imaging plane, as could be seen in Figs. 5 and 6. Radiation doses that differ to much from the optimum, needed by the photoresist, do cause either under- (for to small) or overexposure (for to large). In the case of underexposure, the effect on the master is delamination (in extreme cases) or shrinking of the developed structures. In case of overexposure, the structures will grow over the edges, defined by the photomask. The radiation dose depends of the local light intensity and the overall exposure-time. These two parameters have to be optimized in the process to achieve optimum results for accuracy over a maximized imaging area.

Figure 5 shows the light intensity measured by the camera for different optical reductions. The black ellipses mark the isolines that represent a drop to $50 \%$ light intensity in relation to the image center and the maximum light intensity. The illuminated spot takes a slightly elliptical shape because of the square $2 / 3$ cross-section of the LED chip. Using the proposed setup, the light intensity changes over the image
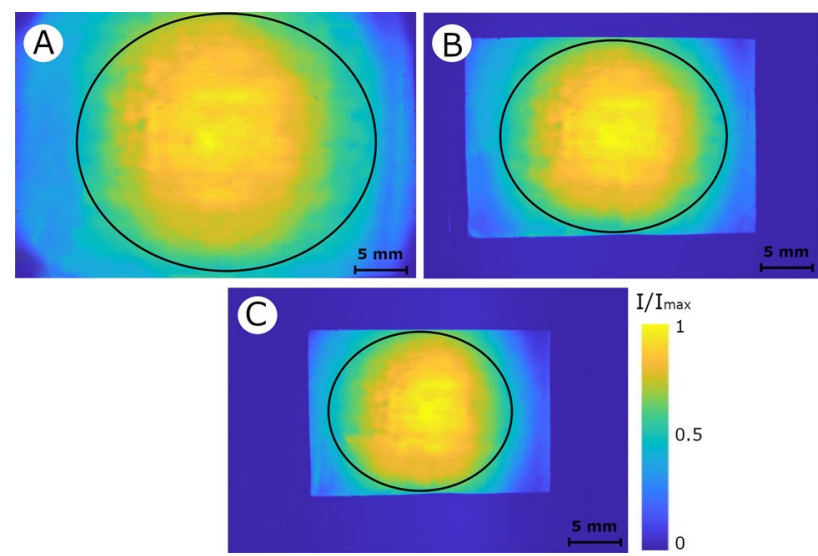

Fig. 5 Relative light intensity at imaging plane (on the substrate) over the image cross-section at $7 \times($ Subimage A), $10 \times($ Subimage B $), 12 \times$ (Subimage C) optical reduction. The solid black ellipse marks the isoline of $50 \%$ relative light intensity

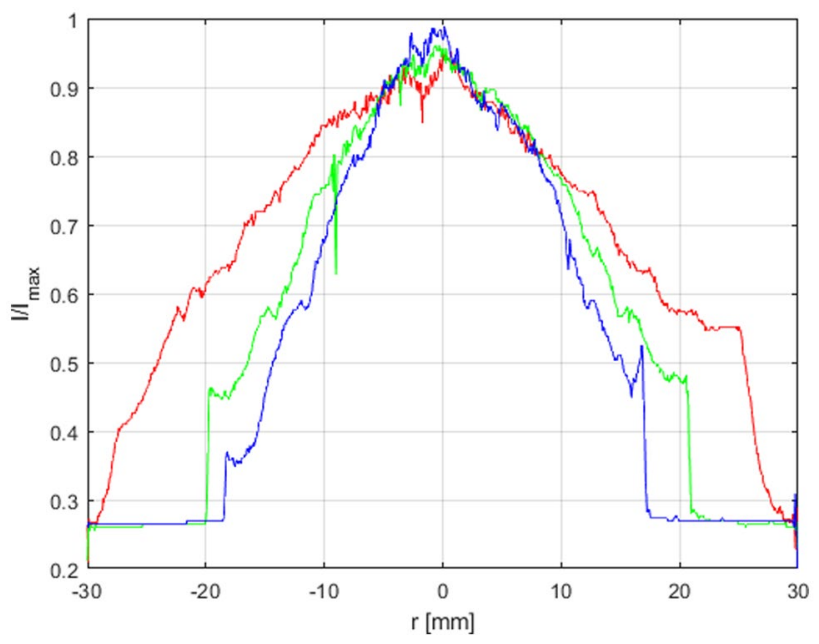

Fig. 6 Relative light intensity over horizontal center line: $(-) 7 \times,(-) 10 \times,(-) 12 \times$

always lead to overexposure in the center region and underexposure in the outer regions of the image.

Figure 7 shows the imaged mask pattern for $10 \times$ optical reduction. The non-uniform illumination is clearly visible in the overall image (Fig. 7, subfigure 1). Two smaller crosssections are displayed in Fig. 7 subfigures 2 and 3, representing the center region and the outer region at $I / I_{\max }=0.5$. The diagram on the right shows the intensity profile over the lines displayed in subfigure 2 and 3 . Generally, the imaging quality, such as edge sharpness and light intensity decreases to the outward regions of the image. Additionally, the light intensities of small structures (left peak) are also decreased. For the center patch (subfigure 3 ) the smaller grids intensity is reduced by $10 \%$ compared to the larger grid. This tendency becomes even more severe at the outer regions (red line, Fig. 7). Here, the intensity drops by approximately 30 $\%$. Also the intensity rise gets less steep with negative impact to the photoresist master. The uneven radiation profile is mainly caused by the radiation profile of the lightsource itself and the losses due to reflection at the first surface of the Fresnel lens. The later could be partly overcome by anti reflective coating. The radiation profile of the LED could be improved by the use of additional collimation lenses.

\section{Results}

Figure 8 shows stacked images of the resulting masters at different optical reductions. The images do not cover the total master area, because of the limitations of the used digital microscope. The master contains areas of delamination at the corners of all three sub-images of Fig. 8, where the photoresist does not stick to the substrate due to underexposure, 

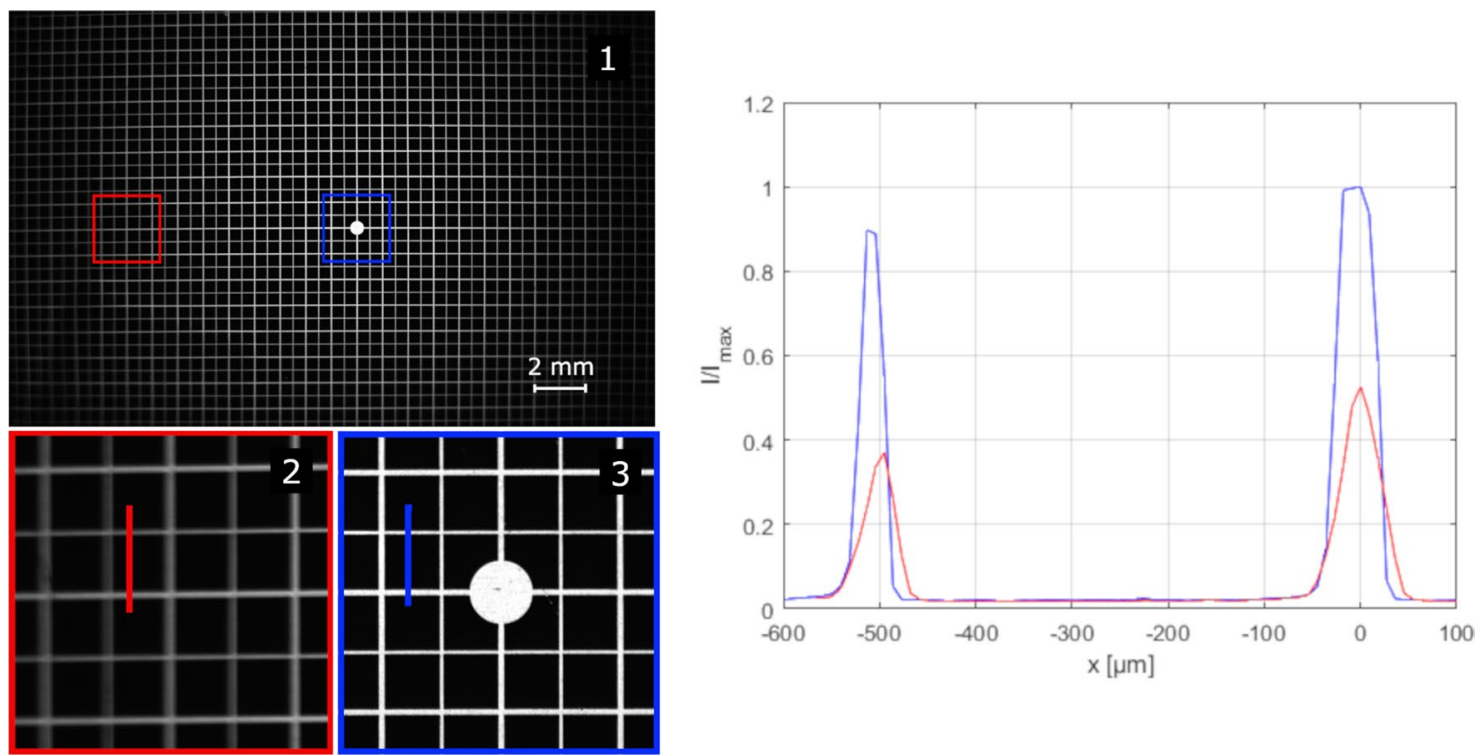

Fig. 7 Imaging quality at $10 \times$ optical reduction
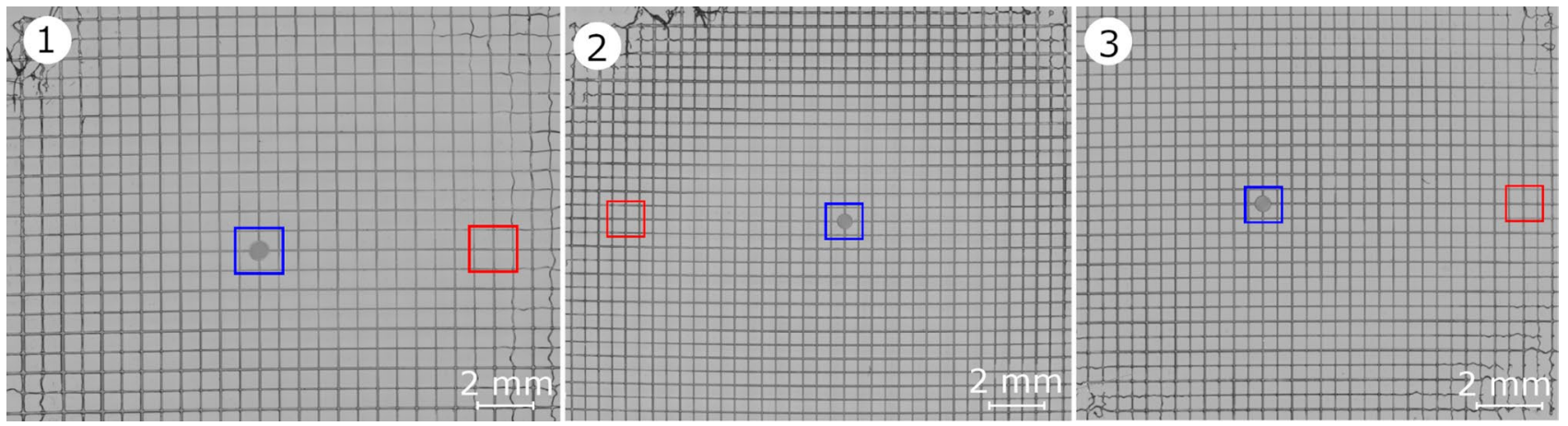

Fig. 8 Grid structures at $7 \times(1), 10 \times(2)$ and $13 \times(3)$ optical reduction. Blue boxes mark grid center, red Boxes mark the outer grid regions $($ color figure online)

rendering the region unusable for the casting of microfluidic structures. The usable mask area increases with increasing optical reduction, while the area of the image is approximately constant. Aside from delaminations, the grids show a slight curvature over the whole master area due to optical distortion and spherical aberrations. Even though imaging errors like astigmatism can be reduced by careful alignment of the optical elements, the severity of optical aberrations mostly depends on the optical elements themselves and their associated apertures. Therefore, the accurate positioning of the optical elements is essential to the setup and optical reduction. However, slight optical distortions do not necessarily affect the applicability of a microfluidic channel, and might therefore be acceptable. Table 1 shows the resulting image (master) sizes by means of their horizontal and vertical dimensions. The master dimensions were measured from the images taken with the digital microscope.
Table 1 Ellipsoidal illuminated mask and image main axis

\begin{tabular}{lllll}
\hline M - & Mask size $h(v)[\mathrm{mm}]$ & Mold $h(v)[\mathrm{mm}]$ & $\begin{array}{l}\operatorname{mi}\left(/ / I_{\max }\right. \\
{[\%]}\end{array}$ & Exposure $[\mathrm{s}]$ \\
\hline $7.5 \times 110(100)$ & $13.3(12.1)$ & 88 & 80 \\
$10 \times$ & $160(135)$ & $13.6(11.5)$ & 88 & 55 \\
$13 \times$ & $160(125)$ & $11.5(8.8)$ & 85 & 40 \\
\hline
\end{tabular}

The applicable mask sizes could be derived by counting the squares in Fig. 8. The image intensities corresponding to the outer region of the master were obtained by the relative intensity curves (Fig. 4) at the locations of the red square centers, drawn in Fig. 8.

By comparing the light intensity profiles and the resulting photoresist masters a minimum relative light intensity of 0.85 could be determined for all optical reductions. This 
limits the achievable master-cross-sections to ellipses with a major axis ratios of 1.2. The necessary exposure times reduced with increasing optical reduction, as in theory the irradiance increases with the negative square of the optical reduction (Kwon and Ju 2018). Optical reductions above 13× have found to be impractical due to low imaging distances between objective lens and substrate. Additionally, at higher optical reductions distance $\mathrm{C}$ has to be increased (see Fig. 1) and therefore the aperture of the imaging optics decreases, resulting in a reduction of the achievable optical resolution. It also becomes increasingly complicated to find the optimal focus for the image.

Figure 9 shows the influence of the exposure time on the resulting grid structure. The subimages in Fig. 9 were taken at the approximate positions of the blue squares in Fig. 8 (subimage 2) for exposure times between 30 and $55 \mathrm{~s}$ at $10 \times$ optical reduction. In general, the exposure time needs to be adjusted for any chosen optical reduction and photoresist thickness. We experienced that the addition of a thin glass plate on the photomask increases the necessary exposure time due to increased light absorption by $20 \%$. As displayed in Fig. 9, low exposure times lead to channel walls with reduced steepness. Especially in the case of $35 \mathrm{~s}$ exposure (Fig. 9, subimage A), the thin grid structure develops a trapezoid shape. Due to the limited resolution of the used transparency photomasks the channel edges are exposed to less radiation, which effects the resolution of the master. This effect is increased, especially for thin structures, in our case at the thin grid structure (vertical channels in Fig. 9). The width of the channel edges decreased from $15 \mu \mathrm{m}$ at $35 \mathrm{~s}$ exposure time to $\sim 4 \mu \mathrm{m}$ at $55 \mathrm{~s}$ exposure time.

Figure 10 shows the center and off-center cross sections marked in Fig. 8 for $7.5 \times, 10 \times$, and $13 \times$ optical reduction. At $7.5 \times$ optical reduction, the photoresist structure is more rough, indicating the influence of imperfections onto the photomask, This might be due to the print quality, but also because of enclosed air bubbles in the acetate film of the used overhead transparencies as pointed out by Love et al. (2001). The channel ledges are $\sim 6 \mu \mathrm{m}$ thick, while the edges onto the photomask are about $40 \mu \mathrm{m}$ thick. This indicates that the mask resolution is also reduced proportionally to the chosen optical reduction. The radius at the crossing of the vertical and horizontal channel was also measured from the images and is $10 \mu \mathrm{m}$.

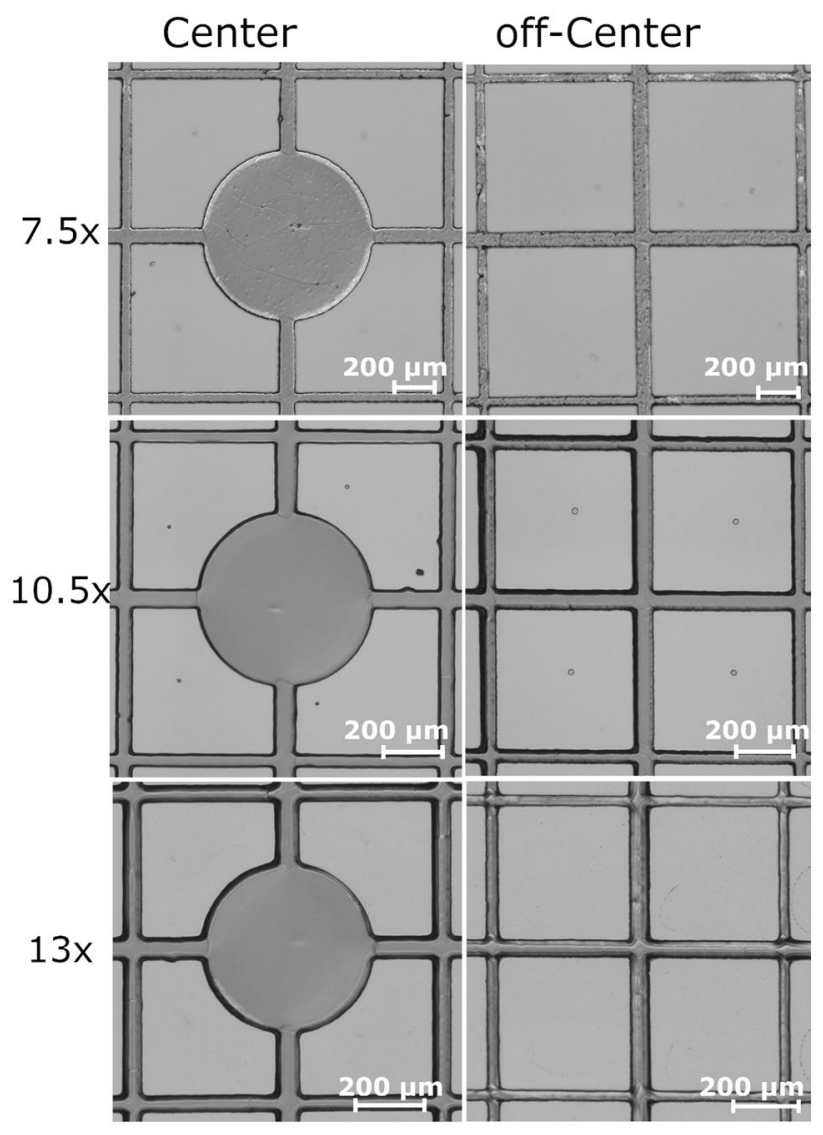

Fig. 10 Detailed images of center- and off-center cross sections marked in Fig. 8 for different optical reductions

The channel widths itself are on average 58 and $32 \mu \mathrm{m}$ for the large and small grid respectively. This is about $20 \%$ smaller than the theoretical channel width calculated from the optical reduction and the channel width on the photomask. At $10 \times$ optical reduction the channel edges are $\sim 4 \mu \mathrm{m}$ wide and there average width is 55 and $28 \mu \mathrm{m}$, respectively. This corresponds to a maximum deviation of $\pm 10 \%$ to the theoretical channel width. For an optical reduction of $13 \times$, the deviations of the channel width increased slightly to $\mp 10 \%$, while the edge width increased significantly to $10 \mu \mathrm{m}$. At the off-center positions the imaging quality generally gets worse because of optical aberrations. The channels tend to have more rounded edges. The deviations from the theoretical channel, however do not exceed $\pm 20 \%$ in width for the small grid structures.
Fig. 9 Variation of exposure time for $10 \times$ optical reduction. A (30 s) B (40 s) C (50 s) D $(55 \mathrm{~s})$
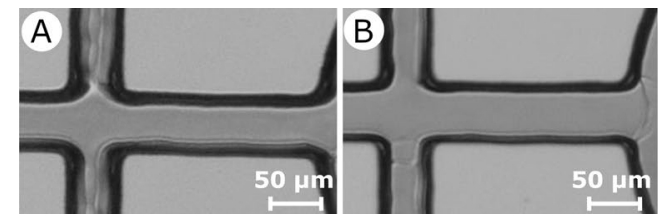

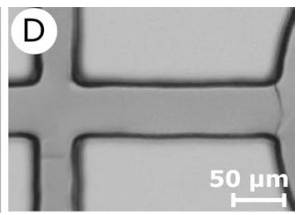




\section{Conclusion}

The present study investigates the applicability of a projection system with optical reduction for the production of microfluidic channels via SU8 based soft lithography. The method, performing at several optical reduction ratios, is able to produce SU8 masters. The achievable accuracy sufficient for structures down to approximately $30 \mu \mathrm{m}$ channel width is mainly limited by the quality of the printed photomasks, as well as increasing optical aberrations and decreasing depth of field for higher optical reduction ratios. However these limitations do not play a critical role in a wide range of microfluidic applications. The authors think that the presented setup could enable researches to rapidly prototype microfluidic geometries without the necessity of high resolution photomasks.

Acknowledgements The authors want to thank the Institute of Physics of the Bundeswehr University Munich and especially Laura Höltgen for the opportunity to use their digital microscope. The Funding of the German Research Foundation (DFG) is gratefully acknowledged. (KA 1808/26-1).

Funding Open Access funding enabled and organized by Projekt DEAL.

Open Access This article is licensed under a Creative Commons Attribution 4.0 International License, which permits use, sharing, adaptation, distribution and reproduction in any medium or format, as long as you give appropriate credit to the original author(s) and the source, provide a link to the Creative Commons licence, and indicate if changes were made. The images or other third party material in this article are included in the article's Creative Commons licence, unless indicated otherwise in a credit line to the material. If material is not included in the article's Creative Commons licence and your intended use is not permitted by statutory regulation or exceeds the permitted use, you will need to obtain permission directly from the copyright holder. To view a copy of this licence, visit http://creativecommons.org/licenses/by/4.0/.

\section{References}

(2019) CBM-120-UVX product datasheet. Luminus Rev 03. https:// download.luminus.com/datasheets/Luminus_CBM-120-UVGen4_Datasheet.pdf

Cabral JT, Hudson SD (2006) Microfluidic approach for rapid multicomponent interfacial tensiometry. Lab Chip 6:427-436

Guillot P, Panizza P, Salmon J-B, Joanicot M, Colin A, Bruneau C-H, Colin T (2006) Viscosimeter on a microfluidic chip. Langmuir 22(14):6438-6445

Hain R, Kähler CJ, Tropea C (2007) Comparison of ccd, cmos and intensified cameras. Exp Fluids 42(3):403-4011

He S, Joseph N, Feng S, Jellicoe M, Raston CL (2020) Application of microfluidic technology in food processing. Food Funct 11:5726-5737

Holmes D, Pettigrew D, Reccius C, Gwyer J, van Berkel C, Holloway J, Davies D, Morgan H (2009) Leukocyte analysis and differentiation using high speed microfluidic single cell impedance cytometry. Lab Chip 9(20):2881-2889

Kwon M, Ju Y-G (2018) Microscope projection photolithography based on ultraviolet light-emitting diodes. Eur J Phy 39(5):055302

Love JC, Wolfe DB, Jacobs HO, Whitesides GM (2001) Microscope projection photolithography for rapid prototyping of masters with micron-scale features for use in soft lithography. Langmuir 17(19):6005-6012

Rothschild M (2005) Projection optical lithography. Mater Today $8(2): 18-24$

Wang T, Quaglio M, Pirri F, Cheng Y-C, Busacker D, Cerrina F (2009) Patterning of SU-8 resist with digital micromirror device (DMD) maskless lithography. In: Levinson HJ, Dusa MV (eds.), Optical Microlithography XXII, volume 7274, pages 857 - 864. International Society for Optics and Photonics, SPIE

Wu K, Zhang H, Chen Y, Luo Q, Xu K (2021) All-silicon microdisplay using efficient hot-carrier electroluminescence in standard 0.18 $m$ cmos technology. IEEE Electron Device Lett 42(4):541-544

Xu K, Chen Y, Okhai TA, Snyman LW (2019) Micro optical sensors based on avalanching silicon light-emitting devices monolithically integrated on chips. Optical Mater Express 9:3985

Publisher's Note Springer Nature remains neutral with regard to jurisdictional claims in published maps and institutional affiliations. 